\title{
Motion Planning for Optimal Information Gathering in Opportunistic Navigation Systems
}

\author{
Zaher M. Kassas*, and Todd E. Humphreys ${ }^{\dagger}$ \\ The University of Texas at Austin, Austin, TX, 78712, USA
}

\begin{abstract}
Motion planning for optimal information gathering in an opportunistic navigation (OpNav) environment is considered. An OpNav environment can be thought of as a radio frequency signal landscape within which a receiver locates itself in space and time by extracting information from ambient signals of opportunity (SOPs). The receiver is assumed to draw only pseudorange-type observations from the SOPs, and such observations are fused through an estimator to produce an estimate of the receiver's own states. Since not all SOP states in the OpNav environment may be known a priori, the receiver must estimate the unknown SOP states of interest simultaneously with its own states. In this work, the following problem is studied. A receiver with no a priori knowledge about its own states is dropped in an unknown, yet observable, OpNav environment. Assuming that the receiver can prescribe its own trajectory, what motion planning strategy should the receiver adopt in order to build a high-fidelity map of the OpNav signal landscape, while simultaneously localizing itself within this map in space and time? To answer this question, first, the minimum conditions under which the OpNav environment is fully observable are established, and the need for receiver maneuvering to achieve full observability is highlighted. Then, motivated by the fact that not all trajectories a receiver may take in the environment are equally beneficial from an information gathering point of view, a strategy for planning the motion of the receiver is proposed. The strategy is formulated in a coupled estimation and optimal control framework of a gradually identified system, where optimality is defined through various information-theoretic measures. Simulation results are presented to illustrate the improvements gained from adopting the proposed strategy over random and pre-defined receiver trajectories.
\end{abstract}

\section{Introduction}

$\mathrm{T}$ HE weakness of global navigation satellite systems (GNSS) space-based signals makes them easy to block intentionally or accidentally, rendering GNSS insufficient for reliable anytime, anywhere navigation. Motivated by the plenitude of ambient radio frequency signals in GNSS-challenged environments, such as indoors, deep urban canyons, and environments experiencing intentional jamming, a new paradigm termed opportunistic navigation (OpNav), has been proposed. ${ }^{1,2}$ This paradigm aims to extract positioning and timing information from ambient radio frequency signals of opportunity (SOPs). OpNav radio receivers continuously search for opportune signals from which to draw navigation and timing information, employing on-the-fly signal characterization as necessary. In collaborative opportunistic navigation (COpNav), multiple OpNav receivers share information to construct and continuously refine a global signal landscape. ${ }^{3}$

In its most general form, OpNav treats all ambient radio signals as potential SOPs, from conventional GNSS signals to communications signals never intended for use as timing or positioning sources. Each signal's relative timing and frequency offsets, transmit location, and frequency stability, are estimated onthe-fly as necessary, with prior information about these quantities exploited when available. At this level of generality, the OpNav estimation problem is similar to the simultaneous localization and mapping (SLAM) problem in robotics. ${ }^{4,5}$ Both imagine an agent which, starting with incomplete knowledge of its location and surroundings, simultaneously builds a map of its environment and locates itself within that map.

*Graduate Research Assistant, Department of Electrical and Computer Engineering, zkassas@ieee.org

${ }^{\dagger}$ Assistant Professor, Department of Aerospace Engineering and Engineering Mechanics, todd.humphreys@mail.utexas.edu 
In traditional SLAM, the map that gets constructed as the agent (typically a robot) moves through the environment is composed of landmarks - walls, corners, posts, etc. - with associated positions. OpNav extends this concept to radio signals, with SOPs playing the role of landmarks. In contrast to a SLAM environmental map, the OpNav signal landscape is dynamic and more complex. For the simple case of pseudorange-only OpNav, where observables consist solely of signal time-of-arrival measurements, one must estimate, besides the position and velocity of each SOP transmitter's antenna phase center, each SOP's time offset from a reference time base, rate of change of time offset, and a set of parameters that characterize the SOP's reference oscillator stability. Even more SOP parameters are required for an OpNav framework in which both pseudorange and carrier phase measurements are ingested into the estimator. ${ }^{1}$ Of course, in addition to the SOP parameters, the OpNav receiver's own position, velocity, time offset, and time offset rate must be estimated. Metaphorically, the signal landscape map can be thought of as a "jello map", with the jello firmer as the clocks are more stable.

The observability of OpNav environments comprising a receiver with velocity random walk dynamics making pseudorange observations on multiple SOPs was analyzed by Kassas and Humphreys. ${ }^{6}$ This analysis considered several scenarios an OpNav environment may exhibit, and for each scenario the following questions were answered: $(i)$ is the environment observable? and $(i i)$ if the environment is not completely observable, what are the unobservable directions in the state space? This analysis was extended to the case of multiple receivers in a COpNav environment, and the degree of observability, also known as estimability, of the various states in the environment was quantified, with special attention paid to the least and most observable states. ${ }^{7}$ While observability is a Boolean property, i.e. it asserts whether a system is observable or not, it does not specify which trajectory is best for estimability. This is the subject of this paper. To this end, the receiver dynamics is modified so to allow for maneuvering in the form of velocity commands.

Optimizing an observer's path in tracking applications has been the subject of extensive research. ${ }^{8,9,10}$ In such problems, the observer, who has perfect knowledge about its own states, is tracking a stationary or a mobile target through its onboard sensors. The trajectory optimization objective is to prescribe optimal trajectories for the observer to follow to maintain good estimates about the target's states. Such problems are typically formulated in an optimal control framework. In SLAM, the problem of trajectory optimization is more involved, due to the coupling between the localization accuracy and the map quality. Initial SLAM research did not take motion control into account and assumed the robot's path to be predetermined or randomly chosen. Of course, not all trajectories a robot can take will be equally beneficial from a localization and mapping accuracy perspective. The problem of trajectory optimization in SLAM has received considerable attention recently. ${ }^{11,12,13}$

A fundamental challenge in all optimization-based approaches is the choice of a proper optimization metric. To this end, the Shannon and Fisher information measures provide a natural way of quantifying the uncertainty about the receiver's states and the fidelity of the signal landscape. Since the receiver and SOP states may not all be known a priori, the optimal motion planning strategy needs to be performed simultaneously with estimating the unknown states. This dependency of the objective functional on the parameters to be estimated is prevalent in the information-based optimization literature. It is best described by Cochran as: "You tell me the value of $\theta$, and I promise to design the best experiment for estimating $\theta "{ }^{14}$ A key difference between this class of problems and classical optimal control problems is that the cost functional to be minimized is time-varying and unknown, as it depends on the action and sensing strategy that one will take, i.e. the system under consideration is gradually identified.

Optimal receiver motion planning in OpNav environments can be thought of as a hybrid of: $(i)$ optimizing an observer's path in tracking problems and (ii) optimizing the robot's path in SLAM. The similarity to the tracking problems stems from the fact that the SOP's state space is not stationary, which is due to the dynamical nature of the clock error states, making the problem analogous to tracking non-stationary targets. The similarity to the SLAM problems stems from the fact that the receiver's objective is to maximize the information gained about the OpNav environment while simultaneously minimizing the uncertainty about its own state estimates.

This paper makes two contributions. First, the observability of an OpNav environment consisting of a receiver which could command its own maneuvers and multiple SOPs is analyzed. The objective of the observability analysis is twofold: $(i)$ establish the minimum conditions under which the OpNav environment is fully-observable, and ( $i i)$ for scenarios where the OpNav environment is not fully-observable, specify the observable states, if any. It is shown that the minimum a priori knowledge needed about the OpNav environment for full observability is reduced from the case where the receiver is moving according to velocity 
random walk dynamics with no control over its maneuvers. The second contribution is the design of a closed-loop receiver motion planning strategy to optimize the information gathered about the OpNav signal landscape states simultaneously with the receiver's own states. To this end, several information-based measures are compared, namely the $(i)$ D-optimality criterion, which is equivalent to minimizing the volume of the uncertainty ellipsoid, ( $i i)$ A-optimality criterion, which is equivalent to minimizing the average variance of the estimates, and ( iii) E-optimality criterion, which is equivalent to minimizing the length of the largest axis of the uncertainty ellipsoid.

The remainder of this paper is organized as follows. Section II describes the OpNav environment dynamics and observation model. Section III analyzes the observability of various scenarios an OpNav environment may exhibit. Section IV presents a strategy for receiver motion planning for optimal information gathering. Section $V$ presents simulation results to illustrate the effectiveness of the proposed strategy. Concluding remarks are given in Section VI.

\section{Model Description}

\section{A. Dynamics Model}

In a generic coordinate $\xi$, the receiver's dynamics will be assumed to evolve according to

$$
\dot{\xi}(t)=u_{\xi}(t)+\tilde{w}_{\xi}(t),
$$

where $u_{\xi}$ is the control input in the form of a velocity command and $\tilde{w}_{\xi}$ is the process noise, which is modeled as a zero-mean white stochastic process with power spectral density $\tilde{q}_{\xi}$, i.e.

$$
\mathbb{E}\left[\tilde{w}_{\xi}(t)\right]=0, \quad \mathbb{E}\left[\tilde{w}_{\xi}(t) \tilde{w}_{\xi}(\tau)\right]=\tilde{q}_{\xi} \delta(t-\tau),
$$

where $\delta(t)$ is the Dirac delta function. Note that in the absence of the control input, $u_{\xi}$, this model is simply position random walk dynamics.

The receiver and SOP clock error dynamics will be modeled according to the so-called two-state model, composed of the clock bias $\delta t$ and clock drift $\dot{\delta} t$. The clock error states evolve according to

$$
\dot{\boldsymbol{x}}_{\mathrm{clk}}(t)=\mathbf{A}_{\mathrm{clk}} \boldsymbol{x}_{\mathrm{clk}}(t)+\tilde{\boldsymbol{w}}_{\mathrm{clk}}(t),
$$

where

$$
\boldsymbol{x}_{\mathrm{clk}}=\left[\begin{array}{c}
\delta t \\
\dot{\delta t}
\end{array}\right], \quad \tilde{\boldsymbol{w}}_{\mathrm{clk}}=\left[\begin{array}{c}
\tilde{w}_{\delta t} \\
\tilde{w}_{\dot{\delta} t}
\end{array}\right], \quad \mathbf{A}_{\mathrm{clk}}=\left[\begin{array}{cc}
0 & 1 \\
0 & 0
\end{array}\right],
$$

where $\tilde{w}_{\delta t}$ and $\tilde{w}_{\dot{\delta} t}$ are zero-mean, mutually independent white noise processes with power spectra $S_{\tilde{w}_{\delta t}}$ and $S_{\tilde{w}_{\delta t}}$, respectively. The power spectra $S_{\tilde{w}_{\delta t}}$ and $S_{\tilde{w}_{\delta t}}$ can be related to the power-law coefficients, $\left\{h_{\alpha}\right\}_{\alpha=-2}^{2}$, which typically characterize the power spectral density of the fractional frequency deviation $y(t)$ of an oscillator from nominal frequency, namely $S_{y}=\sum_{\alpha=-2}^{2} h_{\alpha} f^{\alpha} \cdot{ }^{15}$ It is common to approximate such relationships by considering only the frequency random walk coefficient $h_{-2}$ and the white frequency coefficient $h_{0}$, which lead to $S_{\tilde{w}_{\delta t}} \approx \frac{h_{0}}{2}$ and $S_{\tilde{w}_{\delta t}} \approx 2 \pi^{2} h_{-2} \cdot{ }^{16,17}$

The receiver's state vector will be defined by augmenting the receiver's planar position states with its clock error states to yield the state-space realization

$$
\dot{\boldsymbol{x}}_{r}(t)=\mathbf{A}_{r} \boldsymbol{x}_{r}(t)+\mathbf{B}_{r} \boldsymbol{u}_{r}(t)+\mathbf{D}_{r} \tilde{\boldsymbol{w}}_{r}(t),
$$

where $\boldsymbol{x}_{r}=\left[\boldsymbol{r}_{r}^{\top}, \delta t_{r}, \dot{\delta} t_{r}\right]^{\top}, \boldsymbol{r}_{r}=\left[x_{r}, y_{r}\right]^{\top}, \boldsymbol{u}_{r}=\left[u_{x}, u_{y}\right]^{\top}, \tilde{\boldsymbol{w}}_{r}=\left[\tilde{w}_{x}, \tilde{w}_{y}, \tilde{w}_{\delta t_{r}}, \tilde{w}_{\dot{\delta} t_{r}}\right]^{\top}$

$$
\mathbf{A}_{r}=\left[\begin{array}{ll}
\mathbf{0}_{2 \times 2} & \mathbf{0}_{2 \times 2} \\
\mathbf{0}_{2 \times 2} & \mathbf{A}_{\mathrm{clk}}
\end{array}\right], \quad \mathbf{B}_{r}=\left[\begin{array}{c}
\mathbf{I}_{2 \times 2} \\
\mathbf{0}_{2 \times 2}
\end{array}\right], \quad \mathbf{D}_{r}=\mathbf{I}_{4 \times 4} .
$$

The receiver's dynamics in (1) is discretized at a sampling period $T \triangleq t_{k+1}-t_{k}$, assuming zero-order hold of the control inputs, i.e. $\left\{u(t)=u\left(t_{k}\right), t_{k} \leq t<t_{k+1}\right\}$, to yield the discrete-time (DT) equivalent model

$$
\boldsymbol{x}_{r}\left(t_{k+1}\right)=\mathbf{F}_{r} \boldsymbol{x}_{r}\left(t_{k}\right)+\mathbf{G}_{r} \boldsymbol{u}_{r}\left(t_{k}\right)+\boldsymbol{w}_{r}\left(t_{k}\right), \quad k=0,1,2, \ldots
$$


where $\boldsymbol{w}_{r}$ is a DT zero-mean white noise sequence with covariance $\mathbf{Q}_{r}$, with

$$
\begin{gathered}
\mathbf{F}_{r}=\left[\begin{array}{cc}
\mathbf{I}_{2 \times 2} & \mathbf{0}_{2 \times 2} \\
\mathbf{0}_{2 \times 2} & \mathbf{F}_{\mathrm{clk}}
\end{array}\right], \quad \mathbf{G}_{r}=\left[\begin{array}{c}
T \mathbf{I}_{2 \times 2} \\
\mathbf{0}_{2 \times 2}
\end{array}\right], \quad \mathbf{F}_{\mathrm{clk}}=\left[\begin{array}{cc}
1 & T \\
0 & 1
\end{array}\right] \\
\mathbf{Q}_{r}=\operatorname{diag}\left[\mathbf{Q}_{p}, \mathbf{Q}_{\mathrm{clk}, r}\right], \quad \mathbf{Q}_{p}=\left[\begin{array}{cc}
T \tilde{q}_{x} & 0 \\
0 & T \tilde{q}_{y}
\end{array}\right], \mathbf{Q}_{\mathrm{clk}, r}=\left[\begin{array}{cc}
S_{\tilde{w}_{\delta t_{r}}} T+S_{\tilde{w}_{\dot{\delta} t_{r}}} \frac{T^{3}}{S_{\tilde{w}_{\delta t_{r}}}} & \frac{T^{2}}{2} \\
S_{\tilde{w}_{\dot{\delta} t_{r}}} \frac{T^{2}}{2} & S_{\tilde{w}_{\delta t_{r}}} T
\end{array}\right] .
\end{gathered}
$$

The SOP will be assumed to emanate from a spatially-stationary terrestrial transmitter and its state consists of its planar position and clock error states. Hence, the SOP's dynamics can be described by the state-space model

$$
\dot{\boldsymbol{x}}_{s}(t)=\mathbf{A}_{s} \boldsymbol{x}_{s}(t)+\mathbf{D}_{s} \tilde{\boldsymbol{w}}_{s}(t),
$$

where $\boldsymbol{x}_{s}=\left[\boldsymbol{r}_{s}^{\top}, \delta t_{s}, \dot{\delta t_{s}}\right]^{\top}, \boldsymbol{r}_{s}=\left[x_{s}, y_{s}\right]^{\top}, \boldsymbol{w}_{s}=\left[\tilde{w}_{\delta t_{s}}, \tilde{w}_{\dot{\delta} t_{s}}\right]^{\top}$

$$
\mathbf{A}_{s}=\left[\begin{array}{cc}
\mathbf{0}_{2 \times 2} & \mathbf{0}_{2 \times 2} \\
\mathbf{0}_{2 \times 2} & \mathbf{A}_{\mathrm{clk}}
\end{array}\right], \quad \mathbf{D}_{s}=\left[\begin{array}{c}
\mathbf{0}_{2 \times 2} \\
\mathbf{I}_{2 \times 2}
\end{array}\right]
$$

Discretizing the SOP's dynamics (3) at a sampling interval $T$ yields the DT-equivalent model

$$
\boldsymbol{x}_{s}\left(t_{k+1}\right)=\mathbf{F}_{s} \boldsymbol{x}_{s}\left(t_{k}\right)+\boldsymbol{w}_{s}\left(t_{k}\right),
$$

where $\boldsymbol{w}_{s}$ is a DT zero-mean white noise sequence with covariance $\mathbf{Q}_{s}$, and

$$
\mathbf{F}_{s}=\operatorname{diag}\left[\mathbf{I}_{2 \times 2}, \mathbf{F}_{\mathrm{clk}}\right], \quad \mathbf{Q}_{s}=\operatorname{diag}\left[\mathbf{0}_{2 \times 2}, \mathbf{Q}_{\mathrm{clk}, s}\right]
$$

where $\mathbf{Q}_{\mathrm{clk}, s}$ is identical to $\mathbf{Q}_{\mathrm{clk}, r}$, except that the spectra $S_{\tilde{w}_{\delta t_{r}}}$ and $S_{\tilde{w}_{\dot{\delta} t_{r}}}$ are now replaced with SOP-specific spectra, $S_{\tilde{w}_{\delta t_{s}}}$ and $S_{\tilde{w}_{\delta t_{s}}}$, respectively.

Defining the augmented state as $\boldsymbol{x} \triangleq\left[\boldsymbol{x}_{r}^{\top}, \boldsymbol{x}_{s}^{\top}\right]^{\top}$ and the augmented process noise vector as $\boldsymbol{w} \triangleq$ $\left[\boldsymbol{w}_{r}^{\top}, \boldsymbol{w}_{s}^{\top}\right]^{\top}$ yields the system dynamics

$$
\boldsymbol{x}\left(t_{k+1}\right)=\mathbf{F} \boldsymbol{x}\left(t_{k}\right)+\boldsymbol{w}\left(t_{k}\right)
$$

where $\mathbf{F}=\operatorname{diag}\left[\mathbf{F}_{r}, \mathbf{F}_{s}\right]$, and $\boldsymbol{w}$ is a zero-mean white noise sequence with covariance $\mathbf{Q}=\operatorname{diag}\left[\mathbf{Q}_{r}, \mathbf{Q}_{s}\right]$. While the model defined in (5) considered only one receiver and one SOP, the model can be readily extended to multiple SOPs by augmenting their corresponding states and dynamics.

\section{B. Observation Model}

To properly model the pseudorange observations, one must consider three different time systems. The first is true time, denoted by the variable $t$, which can be considered equivalent to GPS system time. The second time system is that of the receiver's clock and is denoted $t_{r}$. The third time system is that of the SOP's clock and is denoted $t_{s}$. The three time systems are related to each other according to

$$
t=t_{r}-\delta t_{r}(t), \quad t=t_{s}-\delta t_{s}(t)
$$

where $\delta t_{r}(t)$ and $\delta t_{s}(t)$ represent the amount by which the receiver and SOP clocks are different from true time, respectively.

The pseudorange observation made by the receiver on a particular SOP is made in the receiver time and is modeled according to

$\rho\left(t_{r}\right)=\left\|\boldsymbol{r}_{r}\left[t_{r}-\delta t_{r}\left(t_{r}\right)\right]-\boldsymbol{r}_{s}\left[t_{r}-\delta t_{r}\left(t_{r}\right)-\delta t_{\mathrm{TOF}}\right]\right\|_{2}+c \cdot\left\{\delta t_{r}\left(t_{r}\right)-\delta t_{s}\left[t_{r}-\delta t_{r}\left(t_{r}\right)-\delta t_{\mathrm{TOF}}\right]\right\}+\tilde{v}_{\rho}\left(t_{r}\right)$,

where $c$ is the speed of light, $\delta t_{\text {TOF }}$ is the time-of-flight of the signal from the SOP to the receiver, and $\tilde{v}_{\rho}$ is the error in the pseudorange measurement due to modeling and measurement errors. The error $\tilde{v}_{\rho}$ is modeled as a zero-mean white Gaussian noise process with power spectral density $\tilde{r}^{18}$ In $(6)$, the clock offsets $\delta t_{r}$ and $\delta t_{s}$ were assumed to be small and slowly changing, in which case $\delta t_{r}(t)=\delta t_{r}\left[t_{r}-\delta t_{r}(t)\right] \approx \delta t_{r}\left(t_{r}\right)$. The 
first term in (6) is the true range between the receiver's position at time of reception and the SOP's position at time-of-transmission of the signal, while the second term arises due to the offsets from true time in the receiver and SOP clocks.

The observation model in (6) can be further simplified by converting it to true time and invoking mild approximations to arrive at ${ }^{6,7}$

$$
\begin{aligned}
z(t) & =\rho(t) \triangleq h[\boldsymbol{x}(t)]+\tilde{v}_{\rho}(t) \\
& \approx\left\|\boldsymbol{r}_{r}(t)-\boldsymbol{r}_{s}(t)\right\|_{2}+c \cdot\left[\delta t_{r}(t)-\delta t_{s}(t)\right]+\tilde{v}_{\rho}(t)
\end{aligned}
$$

Discretizing the observation equation (7) at a sampling interval $T$ yields the DT-equivalent model

$$
z\left(t_{k}\right)=y\left(t_{k}\right)+v_{\rho}\left(t_{k}\right)=\left\|\boldsymbol{r}_{r}\left(t_{k}\right)-\boldsymbol{r}_{s}\left(t_{k}\right)\right\|_{2}+c \cdot\left[\delta t_{r}\left(t_{k}\right)-\delta t_{s}\left(t_{k}\right)\right]+v_{\rho}\left(t_{k}\right)
$$

where $v_{\rho}$ is a DT zero-mean, white Gaussian process with covariance $r=\tilde{r} / T$.

\section{Observability Analysis}

Conceptually, observability of a dynamic system is a question of solvability of the states from a set of observations that are linearly or nonlinearly related to the states, and where the states evolve according to a set of linear or nonlinear difference or differential equations. Fundamentally, observability of a system is a question of distinguishingly of the initial state from its neighbors. For nonlinear systems, it is more appropriate to analyze the observability through nonlinear observability tools rather than linearizing the nonlinear system and applying linear observability tools. This is due to two reasons: $(i)$ nonlinear observability tools capture the nonlinearities of the dynamics and observations, and ( $i i)$ while the control inputs are never considered in the linear observability tools, they are taken into account in the nonlinear observability tools.

\section{A. Observability of Nonlinear Systems}

For the sake of clarity, the nonlinear observability test employed in this paper is outlined in this subsection. Consider the continuous-time (CT) nonlinear dynamic system in the control affine form, ${ }^{19}$ given by

$$
\Sigma_{\mathrm{NL}}:\left\{\begin{array}{l}
\dot{\boldsymbol{x}}(t)=\boldsymbol{f}_{0}[\boldsymbol{x}(t)]+\sum_{i=1}^{r} \boldsymbol{f}_{i}[\boldsymbol{x}(t)] u_{i}, \quad \boldsymbol{x}\left(t_{0}\right)=\boldsymbol{x}_{0} \\
\boldsymbol{y}(t)=\boldsymbol{h}[\boldsymbol{x}(t)]
\end{array}\right.
$$

where $\boldsymbol{x} \in \mathbb{R}^{n}$ is the system state vector, $\boldsymbol{u} \in \mathbb{R}^{r}$ is the control input vector, $\boldsymbol{y} \in \mathbb{R}^{m}$ is the observation vector, and $\boldsymbol{x}_{0}$ is an arbitrary initial condition.

Two fundamental contrasts between observability of nonlinear and linear systems are: ${ }^{20}$

(i) Choice of inputs. In the linear case, if any input makes the system observable, then every input does so; hence, it suffices to consider the case $\boldsymbol{u} \equiv \mathbf{0}$. In nonlinear systems, this is not the case. Specifically, there may exist certain inputs that could turn an observable system into unobservable. Hence, sensing and actuation in nonlinear systems may be coupled, and they need to be studied simultaneously.

(ii) Length of observations. For observable CT linear systems, observing the outputs $\boldsymbol{y}$ over any arbitrary time interval is sufficient. In nonlinear systems, it may be necessary to observe the outputs over a long, even infinite, time intervals.

Several notions of nonlinear observability exist for $\Sigma_{\mathrm{NL}} \cdot{ }^{21}$ The most general, is (global) nonlinear observability, in which it might be necessary to travel a considerable distance or for a long period of time to distinguish between initial conditions in $\mathbb{R}^{n}$. Observability of $\Sigma_{\mathrm{NL}}$ does not imply that every input $\boldsymbol{u}$ distinguishes initial conditions in $\mathbb{R}^{n}$. Stronger and weaker notions of nonlinear observability exist, namely local observability, weak observability, and local weak observability. A somewhat simple algebraic test exists to assessing local weak observability, which intuitively means that $\boldsymbol{x}_{0}$ is instantaneously distinguishable from its neighbors. This test is based on constructing the so-called nonlinear observability matrix defined next.

Definition 1 The first-order Lie derivative of a scalar function $h$ with respect to a vector-valued function $f$ is defined as

$$
\mathfrak{L}_{\boldsymbol{f}}^{1} h(\boldsymbol{x}) \triangleq \sum_{j=1}^{n} \frac{\partial h(\boldsymbol{x})}{\partial x_{j}} f_{j}(\boldsymbol{x})=\left\langle\nabla_{\boldsymbol{x}} h(\boldsymbol{x}), \boldsymbol{f}(\boldsymbol{x})\right\rangle
$$


where $\boldsymbol{f}(\boldsymbol{x}) \triangleq\left[f_{1}(\boldsymbol{x}), \ldots, f_{n}(\boldsymbol{x})\right]^{\top}$. The zeroth-order Lie derivative of any function is the function itself, i.e. $\mathfrak{L}_{\boldsymbol{f}}^{0} h(\boldsymbol{x})=h(\boldsymbol{x})$. The second-order Lie derivative can be computed recursively as

$$
\mathfrak{L}_{\boldsymbol{f}}^{2} h(\boldsymbol{x})=\mathfrak{L}_{\boldsymbol{f}}\left[\mathfrak{L}_{\boldsymbol{f}}^{1} h(\boldsymbol{x})\right]=\left\langle\left[\nabla_{\boldsymbol{x}} \mathfrak{L}_{\boldsymbol{f}}^{1} h(\boldsymbol{x})\right], \boldsymbol{f}(\boldsymbol{x})\right\rangle .
$$

Higher-order Lie derivatives can be computed similarly. Mixed-order Lie derivatives of $h(\boldsymbol{x})$ with respect to different functions $\boldsymbol{f}_{i}$ and $\boldsymbol{f}_{j}$, given the derivative with respect to $\boldsymbol{f}_{i}$, can be defined as

$$
\mathfrak{L}_{\boldsymbol{f}_{i} \boldsymbol{f}_{j}}^{2} h(\boldsymbol{x}) \triangleq \mathfrak{L}_{\boldsymbol{f}_{j}}^{1}\left[\mathfrak{L}_{\boldsymbol{f}_{i}}^{1} h(\boldsymbol{x})\right]=\left\langle\left[\nabla_{\boldsymbol{x}} \mathfrak{L}_{\boldsymbol{f}_{i}}^{1} h(\boldsymbol{x})\right], \boldsymbol{f}_{j}(\boldsymbol{x})\right\rangle .
$$

The nonlinear observability matrix, denoted $\mathcal{O}_{\mathrm{NL}}$, of $\Sigma_{\mathrm{NL}}$ defined in (9) is a matrix whose rows are the gradients of Lie derivatives, specifically

$$
\mathcal{O}_{\mathrm{NL}} \triangleq\left\{\nabla_{\boldsymbol{x}}^{\top}\left[\mathfrak{L}_{\boldsymbol{f}_{i}, \ldots, \boldsymbol{f}_{j}}^{p} h_{l}(\boldsymbol{x})\right] \mid i, j=0, \ldots, p ; p=0, \ldots, n-1, l=1, \ldots, m\right\},
$$

where $\boldsymbol{h}(\boldsymbol{x}) \triangleq\left[h_{1}(\boldsymbol{x}), \ldots, h_{m}(\boldsymbol{x})\right]^{\top}$.

The significance of $\mathcal{O}_{\mathrm{NL}}$ is that it can be employed to furnish necessary and sufficient conditions for local weak observability. ${ }^{21,22}$ In particular, if $\mathcal{O}_{\mathrm{NL}}$ is full-rank, then $\Sigma_{\mathrm{NL}}$ is said to satisfy the observability rank condition, in which case the system is locally weakly observable. Moreover, if a system $\Sigma_{\mathrm{NL}}$ is locally weakly observable, then the observability rank condition is satisfied generically. The term "generically" means that the observability matrix is full-rank everywhere, except possibly within a subset of the domain of $\boldsymbol{x} .^{20}$

\section{B. Observability Analysis of OpNav Environments}

The observability of various scenarios that may arise in OpNav environments are analyzed in this subsection. The objective of this analysis is twofold: (i) determine whether the environment is observable, and (ii) if the environment is not completely observable, determine the observable states, where the observable states are referred to as those in an orthogonal complement to the unobservable subspace.

\section{Scenarios Overview}

The various scenarios considered in the observability analysis are outlined Table 1 , where $m \in \mathbb{N}$. The first scenario corresponds to a single receiver and a single SOP whose initial states are unknown (no $a$ priori knowledge about any of the states is available). Subsequent scenarios consider cases of partial or full knowledge of initial states. In Table 1, fully-known means that all the initial states are known. Thus, a fully-known receiver is one with known $\boldsymbol{x}_{r}\left(t_{0}\right)$, whereas a fully-known SOP is one with known $\boldsymbol{x}_{s}\left(t_{0}\right)$. On the other hand, partially-known means that only the initial position states are known. Thus, a partially-known receiver is one with known $\boldsymbol{r}_{r}\left(t_{0}\right)$, whereas a partially-known SOP is one with known $\boldsymbol{r}_{s}\left(t_{0}\right)$. For the cases of multiple SOPs, it is assumed that SOPs are not colocated at the same position. Moreover, it is assumed that the receiver identifies the SOPs according to their classification: unknown, partially-known, or fully-known.

Table 1. OpNav observability analysis scenarios considered

\begin{tabular}{|l||l|l|}
\hline Case & Receiver & SOP(s) \\
\hline \hline 1 & 1 Unknown & 1 Unknown \\
\hline 2 & 1 Unknown & $m$ Partially-known \\
\hline 3 & 1 Unknown & 1 Fully-known \\
\hline 4 & 1 Partially-known & 1 Unknown \\
\hline 5 & 1 Partially-known & $m$ Partially-known \\
\hline 6 & 1 Fully-known & 1 Unknown \\
\hline
\end{tabular}




\section{Preliminary Facts}

The following facts will be utilized in assessing the observability of the scenarios in Table 1. The rank of an arbitrary matrix $\mathbf{A} \in \mathbb{R}^{m \times n}$ is the maximal number of linearly independent rows or columns; more specifically, $\operatorname{rank}[\mathbf{A}] \leq \min \{m, n\}$.

The observable states in an OpNav environment, if any, can be found by computing the basis vectors spanning the null space of the nonlinear observability matrix $\mathcal{O}_{\mathrm{NL}}$ and arranging the basis vectors into a matrix. The presence of a row of zeros in such matrix indicates that the corresponding state is observable, since such state is orthogonal to the unobservable subspace.

Having prior knowledge about some of the OpNav environment states is equivalent to augmenting the observation vector with fictitious observations that are identical to such known states. For instance, an environment with a partially-known receiver and an unknown SOP has an observation vector $\boldsymbol{y}=\left[x_{r}, y_{r}, \rho\right]^{\top}$.

Consider an OpNav environment with one receiver and one SOP. From Section II, it can be seen that the vectors $\left\{\boldsymbol{f}_{i}\right\}_{i=0}^{r}$ corresponding to $\Sigma_{\mathrm{NL}}$ in (9) have the form

$$
f_{0}=\dot{\delta} t_{r} e_{3}+\dot{\delta} t_{s} e_{7}, \quad f_{1}=e_{1}, \quad f_{2}=e_{2}
$$

where $\boldsymbol{e}_{i}$ is the standard basis vector consisting of a 1 in the $i$ th element and zeros elsewhere. Consider the vector $\boldsymbol{h}=\left[x_{r}, y_{r}, \delta t_{r}, \dot{\delta} t_{r}, x_{s}, y_{s}, \delta t_{s}, \dot{\delta t_{s}}, \rho\right]^{\top}$.

It can be readily shown that the first-order Lie derivatives of $\left\{h_{l}(\boldsymbol{x})\right\}_{l=1}^{9}$ with respect to $\boldsymbol{f}_{i}$ is given by

$$
\begin{aligned}
& \mathfrak{L}_{\boldsymbol{f}_{0}}^{1} h_{l}(\boldsymbol{x})= \begin{array}{ll}
\dot{\delta} t_{r}, & l=3 ; \\
\dot{\delta} t_{s}, & l=7 ; \\
c \cdot\left(\dot{\delta} t_{r}-\dot{\delta} t_{s}\right) & l=9 ; \\
0, & \text { otherwise }
\end{array} \quad \mathfrak{L}_{\boldsymbol{f}_{1}}^{1} h_{l}(\boldsymbol{x})=\left\{\begin{array}{ll}
1, & l=1 ; \\
g_{1}\left(\boldsymbol{r}_{r}, \boldsymbol{r}_{s}\right), & l=9 ; \\
0, & \text { otherwise }
\end{array} \quad \mathfrak{L}_{\boldsymbol{f}_{2}}^{1} h_{l}(\boldsymbol{x})= \begin{cases}1, & l=2 ; \\
g_{2}\left(\boldsymbol{r}_{r}, \boldsymbol{r}_{s}\right), & l=9 ; \\
0, & \text { otherwise }\end{cases} \right. \\
& g_{1}\left(\boldsymbol{r}_{r}, \boldsymbol{r}_{s}\right) \triangleq \frac{x_{r}-x_{s}}{\sqrt{\left(x_{r}-x_{s}\right)^{2}+\left(y_{r}-y_{s}\right)^{2}}}, \quad g_{2}\left(\boldsymbol{r}_{r}, \boldsymbol{r}_{s}\right) \triangleq \frac{y_{r}-y_{s}}{\sqrt{\left(x_{r}-x_{s}\right)^{2}+\left(y_{r}-y_{s}\right)^{2}}}
\end{aligned}
$$

The second-order Lie derivatives are given by

$$
\mathfrak{L}_{\boldsymbol{f}_{0}}^{2} h_{l}(\boldsymbol{x})=\mathfrak{L}_{\boldsymbol{f}_{0} \boldsymbol{f}_{1}}^{2} h_{l}(\boldsymbol{x})=\mathfrak{L}_{\boldsymbol{f}_{1} \boldsymbol{f}_{0}}^{2} h_{l}(\boldsymbol{x})=0, \forall l ; \quad \mathfrak{L}_{\boldsymbol{f}_{1}}^{2} h_{l}(\boldsymbol{x})=\left\{\begin{array}{l}
\partial_{x_{r}} g_{1}, l=9 ; \\
0, \quad \text { otherwise, }
\end{array} \mathfrak{L}_{\boldsymbol{f}_{2}}^{2} h_{l}(\boldsymbol{x})= \begin{cases}\partial_{y_{r}} g_{2}, l=9 ; \\
0, & \text { otherwise },\end{cases}\right.
$$

where

$$
\partial_{x_{r}} g_{1} \triangleq \frac{\partial}{\partial x_{r}} g_{1}\left(\boldsymbol{r}_{r}, \boldsymbol{r}_{s}\right), \quad \partial_{y_{r}} g_{2} \triangleq \frac{\partial}{\partial y_{r}} g_{2}\left(\boldsymbol{r}_{r}, \boldsymbol{r}_{s}\right) .
$$

Therefore, the gradients of the Lie derivatives comprising the rows of $\mathcal{O}_{\mathrm{NL}}$ defined in (13) are given by

$$
\begin{aligned}
& \nabla_{\boldsymbol{x}}^{\boldsymbol{\top}}\left[\mathfrak{L}_{\boldsymbol{f}_{i}^{0}} h_{l}(\boldsymbol{x})\right]=\left\{\begin{array}{ll}
g_{1} \cdot\left(\boldsymbol{e}_{1}^{\boldsymbol{\top}}-\boldsymbol{e}_{5}^{\boldsymbol{\top}}\right)+g_{2} \cdot\left(\boldsymbol{e}_{2}^{\boldsymbol{\top}}-\boldsymbol{e}_{6}^{\boldsymbol{\top}}\right)+c \cdot\left(\boldsymbol{e}_{3}^{\boldsymbol{\top}}-\boldsymbol{e}_{7}^{\boldsymbol{\top}}\right), & l=9 ; \\
\boldsymbol{e}_{l}^{\boldsymbol{T}}, & \text { otherwise }
\end{array} \quad \text { for } i=0,1,2\right. \\
& \nabla_{\boldsymbol{x}}^{\top}\left[\mathfrak{L}_{\boldsymbol{f}_{0}}^{1} h_{l}(\boldsymbol{x})\right]= \begin{cases}\boldsymbol{e}_{4}^{\top}, & l=3 ; \\
\boldsymbol{e}_{8}^{\top}, & l=7 ; \\
c \cdot\left(\boldsymbol{e}_{4}^{\top}-\boldsymbol{e}_{8}^{\boldsymbol{\top}}\right), & l=9 ; \\
\mathbf{0}^{\top}, & \text { otherwise. }\end{cases} \\
& \nabla_{\boldsymbol{x}}^{\boldsymbol{\top}}\left[\mathfrak{L}_{\boldsymbol{f}_{i}}^{1} h_{l}(\boldsymbol{x})\right]=\left\{\begin{array}{ll}
\partial_{x_{r}} g_{i} \cdot\left(\boldsymbol{e}_{1}^{\boldsymbol{T}}-\boldsymbol{e}_{5}^{\boldsymbol{\top}}\right)+\partial_{y_{r}} g_{i} \cdot\left(\boldsymbol{e}_{2}^{\top}-\boldsymbol{e}_{6}^{\boldsymbol{\top}}\right), & l=9 ; \\
\mathbf{0}^{\boldsymbol{\top}}, & \text { otherwise; }
\end{array} \quad \text { for } i=1,2\right. \\
& \nabla_{\boldsymbol{x}}^{\boldsymbol{T}}\left[\mathfrak{L}_{\boldsymbol{f}_{0}}^{2} h(\boldsymbol{x})\right]=\nabla_{\boldsymbol{x}}^{\boldsymbol{T}}\left[\mathfrak{L}_{\boldsymbol{f}_{0} \boldsymbol{f}_{1}}^{2} h(\boldsymbol{x})\right]=\nabla_{\boldsymbol{x}}^{\boldsymbol{\top}}\left[\mathfrak{L}_{\boldsymbol{f}_{1} \boldsymbol{f}_{0}}^{2} h(\boldsymbol{x})\right]=\mathbf{0}^{\boldsymbol{\top}}, \\
& \nabla_{\boldsymbol{x}}^{\top}\left[\mathfrak{L}_{\boldsymbol{f}_{i}}^{2} h_{l}(\boldsymbol{x})\right]= \begin{cases}\partial_{x_{r}}\left[\partial_{\alpha_{r}} g_{i}\right] \cdot\left(\boldsymbol{e}_{1}^{\top}-\boldsymbol{e}_{5}^{\boldsymbol{\top}}\right)+\partial_{y_{r}}\left[\partial_{\alpha_{r}} g_{i}\right] \cdot\left(\boldsymbol{e}_{2}^{\top}-\boldsymbol{e}_{6}^{\boldsymbol{\top}}\right), & l=9 ; \\
\mathbf{0}^{\top}, & \text { otherwise; }\end{cases}
\end{aligned}
$$

where $\left\{\alpha=x_{r}\right.$ for $i=1 ; \alpha=y_{r}$ for $\left.i=2\right\}$. 


\section{Observability Analysis}

Case 1. The observation vector has the form $\boldsymbol{y}=[\rho]$ and $\boldsymbol{x} \in \mathbb{R}^{8}$. The only linearly independent rows are those corresponding to $\left\{\nabla_{\boldsymbol{x}}^{\boldsymbol{\top}}\left[\mathfrak{L}_{\boldsymbol{f}_{0}}^{0} h(\boldsymbol{x})\right], \nabla_{\boldsymbol{x}}^{\boldsymbol{\top}}\left[\mathfrak{L}_{\boldsymbol{f}_{0}}^{1} h(\boldsymbol{x})\right], \nabla_{\boldsymbol{x}}^{\top}\left[\mathfrak{L}_{\boldsymbol{f}_{1}}^{1} h(\boldsymbol{x})\right], \nabla_{\boldsymbol{x}}^{\boldsymbol{\top}}\left[\mathfrak{L}_{\boldsymbol{f}_{1}}^{2} h(\boldsymbol{x})\right]\right\}$, and the null space of $\mathcal{O}_{\mathrm{NL}}$ is given by

$$
\mathcal{N}\left[\mathcal{O}_{\mathrm{NL}}\right]=\operatorname{span}\left\{\boldsymbol{e}_{1}+\boldsymbol{e}_{5}, \boldsymbol{e}_{2}+\boldsymbol{e}_{6}, \boldsymbol{e}_{3}+\boldsymbol{e}_{7}, \boldsymbol{e}_{4}+\boldsymbol{e}_{8}\right\} .
$$

Hence, the environment is unobservable and none of the states is observable.

Case 2. The observation vector has the form $\boldsymbol{y}=\left[x_{s_{1}}, y_{s_{1}}, \ldots, x_{s_{m}}, y_{s_{m}}, \rho_{s_{1}}, \ldots, \rho_{s_{m}}\right]$, where $\left\{\rho_{s_{i}}\right\}_{i=1}^{m}$ is the pseudorange between the receiver and SOP $i$, and $\boldsymbol{x} \in \mathbb{R}^{4+4 m}$. The only linearly independent rows are those corresponding to

$$
\left\{\nabla_{\boldsymbol{x}}^{\boldsymbol{\top}}\left[\mathfrak{L}_{\boldsymbol{f}_{0}}^{0} h_{l}(\boldsymbol{x})\right], \nabla_{\boldsymbol{x}}^{\boldsymbol{\top}}\left[\mathfrak{L}_{\boldsymbol{f}_{0}}^{1} h_{3 m-(i-1)}(\boldsymbol{x})\right], \nabla_{\boldsymbol{x}}^{\boldsymbol{\top}}\left[\mathfrak{L}_{\boldsymbol{f}_{1}}^{1} h_{3 m-(i-1)}(\boldsymbol{x})\right], \nabla_{\boldsymbol{x}}^{\boldsymbol{\top}}\left[\mathfrak{L}_{\boldsymbol{f}_{1}}^{2} h_{3 m-(i-1)}(\boldsymbol{x})\right]\right\}
$$

where $l=1, \ldots, 3 m, i=1, \ldots, m$, and the null space of $\mathcal{O}_{\mathrm{NL}}$ is given by

$$
\mathcal{N}\left[\mathcal{O}_{\mathrm{NL}}\right]=\operatorname{span}\left\{\boldsymbol{e}_{3}+\sum_{i=1}^{m} \boldsymbol{e}_{3+4 i}, \boldsymbol{e}_{4}+\sum_{i=1}^{m} \boldsymbol{e}_{4+4 i}\right\} .
$$

Hence, the environment is unobservable, but the position states of the receiver, $x_{r}$ and $y_{r}$, are observable, along with the position states of the SOPs, $x_{s_{i}}$ and $y_{s_{i}}$, which are observable by construction.

Case 3. The observation vector has the form $\boldsymbol{y}=\left[x_{s}, y_{s}, \delta t_{s}, \dot{\delta} t_{s}, \rho\right]$ and $\boldsymbol{x} \in \mathbb{R}^{8}$. The only linearly independent rows are those corresponding to $\left\{\nabla_{\boldsymbol{x}}^{\top}\left[\mathfrak{L}_{\boldsymbol{f}_{0}}^{0} h_{l}(\boldsymbol{x})\right], \nabla_{\boldsymbol{x}}^{\top}\left[\mathfrak{L}_{\boldsymbol{f}_{0}}^{1} h_{5}(\boldsymbol{x})\right], \nabla_{\boldsymbol{x}}^{\top}\left[\mathfrak{L}_{\boldsymbol{f}_{1}}^{1} h_{5}(\boldsymbol{x})\right], \nabla_{\boldsymbol{x}}^{\boldsymbol{T}}\left[\mathfrak{L}_{\boldsymbol{f}_{1}}^{2} h_{5}(\boldsymbol{x})\right]\right\}$, where $l=1, \ldots, 5$. Hence, $\mathcal{O}_{\mathrm{NL}}$ is full-rank, and the environment is fully-observable.

Case 4. The observation vector has the form $\boldsymbol{y}=\left[x_{r}, y_{r}, \rho\right]$ and $\boldsymbol{x} \in \mathbb{R}^{8}$. The only linearly independent rows are those corresponding to $\left\{\nabla_{\boldsymbol{x}}^{\boldsymbol{T}}\left[\mathfrak{L}_{\boldsymbol{f}_{0}}^{0} h_{l}(\boldsymbol{x})\right], \nabla_{\boldsymbol{x}}^{\boldsymbol{T}}\left[\mathfrak{L}_{\boldsymbol{f}_{0}}^{1} h_{3}(\boldsymbol{x})\right], \nabla_{\boldsymbol{x}}^{\boldsymbol{T}}\left[\mathfrak{L}_{\boldsymbol{f}_{1}}^{1} h_{3}(\boldsymbol{x})\right], \nabla_{\boldsymbol{x}}^{\boldsymbol{T}}\left[\mathfrak{L}_{\boldsymbol{f}_{1}}^{2} h_{3}(\boldsymbol{x})\right]\right\}$, where $l=1,2,3$, and the null space of $\mathcal{O}_{\mathrm{NL}}$ is given by

$$
\mathcal{N}\left[\mathcal{O}_{\mathrm{NL}}\right]=\operatorname{span}\left\{\boldsymbol{e}_{3}+\boldsymbol{e}_{7}, \boldsymbol{e}_{4}+\boldsymbol{e}_{8}\right\}
$$

Hence, the environment is unobservable, but the position states of the SOP, $x_{s}$ and $y_{s}$, are observable, along with the position states of the receiver, $x_{r}$ and $y_{r}$, which are observable by construction.

Case 5. The observation vector has the form $\boldsymbol{y}=\left[x_{r}, y_{r}, x_{s_{1}}, y_{s_{1}}, \ldots, x_{s_{m}}, y_{s_{m}}, \rho_{s_{1}}, \ldots, \rho_{s_{m}}\right]$ and $\boldsymbol{x} \in$ $\mathbb{R}^{4+4 m}$. The only linearly independent rows are $\left\{\nabla_{\boldsymbol{x}}^{\boldsymbol{\top}}\left[\mathfrak{L}_{\boldsymbol{f}_{0}}^{0} h_{l}(\boldsymbol{x})\right], \nabla_{\boldsymbol{x}}^{\boldsymbol{\top}}\left[\mathfrak{L}_{\boldsymbol{f}_{0}}^{1} h_{2+3 m-(i-1)}(\boldsymbol{x})\right]\right\}$, where $l=$ $1,2, \ldots, 2+3 m$ and $i=1, \ldots, m$, and the null space of $\mathcal{O}_{\mathrm{NL}}$ is given by

$$
\mathcal{N}\left[\mathcal{O}_{\mathrm{NL}}\right]=\operatorname{span}\left\{\boldsymbol{e}_{3}+\sum_{i=1}^{m} \boldsymbol{e}_{3+4 i}, \boldsymbol{e}_{4}+\sum_{i=1}^{m} \boldsymbol{e}_{4+4 i}\right\} .
$$

Hence, the environment is unobservable, and none of the states is observable, other than those that observable by construction, namely $x_{r}, y_{r}, x_{s_{i}}$, and $y_{s_{i}}$.

Case 6. The observation vector has the form $\boldsymbol{y}=\left[x_{r}, y_{r}, \delta t_{r}, \dot{\delta} t_{r}, \rho\right]$ and $\boldsymbol{x} \in \mathbb{R}^{8}$. The only linearly independent rows are $\left\{\nabla_{\boldsymbol{x}}^{\boldsymbol{T}}\left[\mathfrak{L}_{\boldsymbol{f}_{0}}^{0} h_{l}(\boldsymbol{x})\right], \nabla_{\boldsymbol{x}}^{\boldsymbol{T}}\left[\mathfrak{L}_{\boldsymbol{f}_{0}}^{1} h_{5}(\boldsymbol{x})\right], \nabla_{\boldsymbol{x}}^{\boldsymbol{T}}\left[\mathfrak{L}_{\boldsymbol{f}_{1}}^{1} h_{5}(\boldsymbol{x})\right], \nabla_{\boldsymbol{x}}^{\boldsymbol{T}}\left[\mathfrak{L}_{\boldsymbol{f}_{1}}^{2} h_{5}(\boldsymbol{x})\right]\right\}$, where $l=$ $1,2, \ldots, 5$. Hence, $\mathcal{O}_{\mathrm{NL}}$ is full-rank, and the environment is fully-observable.

\section{Conclusions:}

1. An OpNav environment with the dynamics and observation models defined in Section II is fully observable if and only if the initial states of at least one SOP are fully-known or the initial states of the receiver are fully-known. 
2. The inputs are necessary to make Cases 3 and 6 observable, since the Lie derivative contributions of $\boldsymbol{f}_{1}$ or $\boldsymbol{f}_{2}$ are needed to make $\mathcal{O}_{\mathrm{NL}}$ full-rank. While the inputs corresponding to $\boldsymbol{f}_{1}$ or $\boldsymbol{f}_{2}$ can be specified in infinitely many ways, the only requirement is that such inputs be non-zero.

3. Adding inputs reduces the a priori knowledge needed for observability of the OpNav environment. Specifically, it was previously shown that an environment comprising a receiver with velocity random walk dynamics and no inputs, making pseudorange observations on multiple SOPs is completely observable if and only if the initial states of at least: $(i)$ one SOP is fully-known and one SOP is partially-known, (ii) the receiver is partially-known and one SOP is fully-known, or (iii) the receiver is fully-known. ${ }^{7}$ In contrast, Case 3 concludes that if the receiver can command its own maneuvers, then conditions $(i)$ and $(i i)$ are reduced as the knowledge of only one fully-known SOP suffices for complete observability, i.e. there is no need for a partially-known SOP nor a partially-known receiver, respectively. Moreover, it was previously shown that for an environment with an unknown receiver with velocity random walk dynamics, at least two partially-known SOPs are needed to make the position states of the receiver observable. ${ }^{7}$ Case 2 concludes that this requirement is reduced to at least one partially-known SOP whenever the receiver is driven by control inputs.

\section{Optimal Motion Planning}

\section{A. Information Optimization Measures}

The observability results in Section III only establish when the OpNav environment is observable, but they do not specify which receiver inputs are best for observability. This section prescribes such inputs. The main objective of the receiver's optimal motion planning is to evaluate different sensing actions that the receiver can take, and choose the action that maximizes the information acquired about the environment, while simultaneously minimizing the uncertainty about the receiver's own states. In this respect, this can be viewed as an adaptive sensing problem, in which one adaptively plans trajectories to maximize the amount or quality of gathered information. ${ }^{11}$

A fundamental challenge in all optimization-based approaches is the choice of a proper optimization metric. In the OpNav context, one needs to quantify the expected acquired information for different sensing actions the receiver may take. To this end, the Shannon entropy and Fisher information measures will be adopted. Broadly, Shannon entropy is related to the volume of a set containing a specified probability mass, while Fisher information is related to the surface area of this set. ${ }^{23}$

A common measure of uncertainty is the notion of entropy, which was introduced by Shannon. Entropy measures the compactness, and thus the informativeness, of a distribution. ${ }^{24}$ The entropy of a random vector $\boldsymbol{x}$ with distribution $p(\boldsymbol{x})$ is defined as

$$
H(\boldsymbol{x}) \triangleq-\int_{\infty}^{\infty} p(\boldsymbol{x}) \log [p(\boldsymbol{x})] d \boldsymbol{x} .
$$

The mutual entropic information loss after an action $\boldsymbol{u}$ is defined as $\Delta I(\boldsymbol{u})=H(\boldsymbol{x} \mid \boldsymbol{u})-H(\boldsymbol{x})$, where $H(\boldsymbol{x} \mid \boldsymbol{u})$ is the conditional entropy after action $\boldsymbol{u}$. A multi-variate Gaussian random vector $\boldsymbol{x}$ has entropy proportional to the logarithm of the determinant of its covariance matrix $\mathbf{P}$, namely $H(\boldsymbol{x})=\frac{1}{2} \log \left[(2 \pi e)^{n} \operatorname{det}(\mathbf{P})\right]$. Therefore, for a Gaussian random vector $\boldsymbol{x}\left(t_{k}\right)$ with covariance $\mathbf{P}\left(t_{k}\right)$, it can be shown that to minimize the mutual entropic information loss after an action $\boldsymbol{u}\left(t_{k}\right)$, one needs to solve the optimization problem

$$
\underset{\boldsymbol{u}\left(t_{k}\right)}{\operatorname{maximize}} \log \operatorname{det}\left[\frac{\mathbf{Y}\left[t_{k+1} \mid \boldsymbol{u}\left(t_{k}\right)\right]}{\mathbf{Y}\left(t_{k}\right)}\right],
$$

where $\mathbf{Y}\left(t_{k}\right) \triangleq \mathbf{P}^{-1}\left(t_{k}\right)$ is the information matrix and $\mathbf{Y}\left[t_{k+1} \mid \boldsymbol{u}\left(t_{k}\right)\right]$ is the corresponding information matrix after action $\boldsymbol{u}(k)$. Recognizing that $\mathbf{Y}\left(t_{k}\right)$ corresponds to the Fisher information matrix, one can establish the connection between the Shannon entropy and the Fisher information: minimization of Shannon entropy is equivalent to maximization of Fisher information. This is the basis of the so-called D-optimality criterion.

Given an information matrix, Y, some of the most common optimization matrix measures are ${ }^{25}$

D-optimality: is equivalent to minimization of the volume of the uncertainty ellipsoid and is given by

$$
\text { minimize } \mathcal{J}=-\log \operatorname{det}[\mathbf{Y}]
$$


A-optimality: is equivalent to minimization of the the average variance of the estimates and is given by

$$
\text { minimize } \mathcal{J}=\operatorname{tr}\left[\mathbf{Y}^{-1}\right]
$$

E-optimality: is equivalent to minimization of the length of the largest axis of the uncertainty ellipsoid and is given by

$$
\operatorname{minimize} \mathcal{J}=\lambda_{\max }\left[\mathbf{Y}^{-1}\right]
$$

where $\lambda_{\max }$ is the largest eigenvalue.

\section{B. Optimal Receiver Motion Planning Strategy}

A unique feature of OpNav is that the quality of the estimates not only depends on the spatial trajectory the receiver takes within the environment, but also depends on the velocity with which the receiver traverses such trajectory. This can be explained by examining the pseudorange observation model derived in (8). Note the term due to the clock biases $c \cdot\left[\delta t_{r}\left(t_{k}\right)-\delta t_{s}\left(t_{k}\right)\right]$, and recall the two state model governing the evolution of the clock bias and drift states over time, which is essentially a double integrator driven by exogenous stochastic processes. Hence, the state space of each SOP contains time-invariant (static) states, $x_{s}$ and $y_{s}$, along with time-varying (dynamic) states, $\delta t_{s}$ and $\dot{\delta} t_{s}$. This makes the estimation problem similar to that of observers tracking dynamic targets, to which the velocity of the observer (tracker) affects the quality of the estimates. The effect of the receiver velocity on the quality of estimates can also be seen by considering a time history of $N$ observations $Z^{N} \triangleq\left\{z\left(t_{1}\right), z\left(t_{2}\right), \ldots, z\left(t_{N}\right)\right\}$, collected at a sampling period $T$, by a receiver that traversed a particular trajectory at a speed $s$. If the receiver doubled its speed, i.e. to become $2 s$, it could have collected the same number of observations at half the sampling period $T / 2$. Recall that the covariance of the process noise characterizing the clock bias and drift noise $\mathbf{Q}_{\mathrm{clk}}$ is a function of $T, T^{2}$, and $T^{3}$. Therefore, reducing $T$ effectively reduces $\mathbf{Q}_{\mathrm{clk}}$, which in turn reduces the estimation error.

The proposed optimal receiver motion planning loop is depicted in Figure 1. At a particular time step $t_{k}$, the pseudorange observations made by the receiver on the various SOPs in the environment, $\boldsymbol{z}\left(t_{k}\right) \triangleq$ $\left[z_{1}\left(t_{k}\right), \ldots, z_{m}\left(t_{k}\right)\right]^{\top}$, are fused through an estimator, an extended Kalman filter (EKF) in this case, which produces state estimate $\hat{\boldsymbol{x}}\left(t_{k} \mid t_{k}\right)$ and associated estimation error covariance $\mathbf{P}\left(t_{k} \mid t_{k}\right)$. The estimate and associated covariance are fed to an optimizer, which solves for the optimal admissible control input $\boldsymbol{u}^{\star}\left(t_{k}\right)$ that is the solution to the constrained nonlinear optimization problem

$$
\begin{array}{cl}
\underset{\boldsymbol{u}\left(t_{k}\right)}{\operatorname{minimize}} & \mathcal{J}\left[\boldsymbol{u}\left(t_{k}\right)\right] \\
\text { subject to } & \boldsymbol{x}_{r}\left(t_{k+1}\right)=\mathbf{F}_{r} \boldsymbol{x}_{r}\left(t_{k}\right)+\mathbf{G}_{r} \boldsymbol{u}\left(t_{k}\right)+\boldsymbol{w}_{r}\left(t_{k}\right) \\
& \boldsymbol{x}_{s_{i}}\left(t_{k+1}\right)=\mathbf{F}_{s} \boldsymbol{x}_{s_{i}}\left(t_{k}\right)+\boldsymbol{w}_{s_{i}}\left(t_{k}\right) \\
& z_{i}\left(t_{k}\right)=h\left[\boldsymbol{x}_{r}\left(t_{k}\right), \boldsymbol{x}_{s_{i}}\left(t_{k}\right)\right]+\boldsymbol{v}_{i}\left(t_{k}\right), i=1,2, \ldots, m \\
& \left\|\boldsymbol{u}_{r}\left(t_{k}\right)\right\|_{2} \leq u_{r, \max } \\
& \left\|\boldsymbol{u}_{r}\left(t_{k}\right)-\boldsymbol{u}_{r}^{\star}\left(t_{k-1}\right)\right\|_{2} \leq T a_{r, \max },
\end{array}
$$

where $u_{r, \max }$ and $a_{r, \max }$ are the maximum speed and acceleration, respectively, with which the receiver can move. Note that the optimization variable is $\boldsymbol{u}_{r}(k)$, whereas $\boldsymbol{u}_{r}^{\star}(k-1)$ is a known constant vector representing the velocity commands that resulted from solving the optimization problem in the previous time-step $k-1$ and has already been applied. The cost functional $\mathcal{J}\left[\boldsymbol{u}\left(t_{k}\right)\right]$ is the D-optimality, A-optimality, or E-optimality criterion, where $\mathbf{Y}=\mathbf{P}^{-1}\left(t_{k+1} \mid t_{k+1}\right)$, i.e. the inverse of the updated estimation error covariance computed by the EKF. It is worth noting that $\mathbf{P}\left(t_{k+1} \mid t_{k+1}\right)$ is a function of $\boldsymbol{u}\left(t_{k}\right)$ and can be computed without knowledge of the next observation $\boldsymbol{z}\left(t_{k+1}\right)$. The optimal control input $\boldsymbol{u}^{\star}\left(t_{k}\right)$ is fed-back to the receiver to command its velocity and is also communicated with the estimator. The produced control $\boldsymbol{u}^{\star}\left(t_{k}\right)$ is commonly referred to as the local or greedy optimal control, since it is based on a single-step look-ahead.

In principle, the above optimization problem can be solved analytically using Lagrange multipliers. However, the problem becomes quickly intractable as more SOPs are present in the environment. In particular, the difficulty stems from the matrix inversion step in the EKF algorithm. In this paper, numerical methods were employed to solve the constrained nonlinear optimization problem via the sequential quadratic programming (SQP) method. 


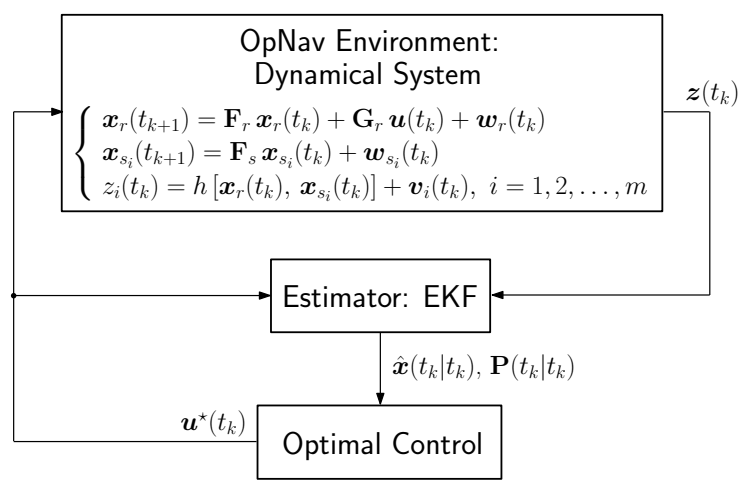

Figure 1. Optimal receiver motion planning loop

\section{Simulation Results}

Consider the following scenario. A receiver that could control its own maneuvers, but with no a priori knowledge about its own states is dropped in an unknown signal landscape with one "anchor" SOP whose initial states are known. Recall from Section III that such scenario is observable. The receiver's objective is to build as quickly as possible a high-fidelity map of the signal landscape states, while simultaneously localizing itself within this map in space and time with high accuracy. This section presents simulation results for five receiver trajectories: a random trajectory in which the receiver velocity commands are chosen randomly but are known to the receiver, a pre-defined circular trajectory around the known anchor SOP, and optimal trajectories as computed by the D-optimality, A-optimality, and E-optimality criteria.

For purposes of numerical stability, the clock error states were defined to be $c \delta t$ and $c \dot{\delta} t$. The environment consisted of a receiver with an initial state vector $\boldsymbol{x}_{r}\left(t_{0}\right)=[0,0,100,10]^{\top}$, an anchor SOP with an initial state vector $\boldsymbol{x}_{s_{1}}\left(t_{0}\right)=[0,150,10,0.1]^{\top}$, and three unknown SOPs with initial state vectors $\boldsymbol{x}_{s_{2}}\left(t_{0}\right)=[100,-150,20,0.2]^{\top}, \boldsymbol{x}_{s_{3}}\left(t_{0}\right)=[200,200,30,0.3]^{\top}, \boldsymbol{x}_{s_{4}}\left(t_{0}\right)=[-150,50,40,0.4]^{\top}$, respectively. The receiver's clock was assumed to be a temperature-compensated crystal oscillator (TCXO) with $h_{0}=2 \times 10^{-19}$ and $h_{-2}=2 \times 10^{-20}$, while the SOPs' clocks were assumed to be oven-controlled crystal oscillators (OCXOs) with $h_{0}=8 \times 10^{-20}$ and $h_{-2}=4 \times 10^{-23}$. The process and observation noise spectral densities were taken to be $\tilde{q}_{x}=\tilde{q}_{x}=0.1$ and $\tilde{r}=50$, respectively, and the sampling time was set to $T=0.1$ second. The receiver's maximum speed and acceleration were set to $u_{\max }=\sqrt{200} \mathrm{~m} / \mathrm{s}$ and $a_{\max }=5 \mathrm{~m} / \mathrm{s}^{2}$, respectively. The EKF receiver initial estimates were $\hat{\boldsymbol{x}}_{r}\left(t_{0} \mid t_{0}\right)=[75,75,0,0,]^{\top}$ and SOPs initial estimates were $\hat{\boldsymbol{x}}_{s_{2}}\left(t_{0} \mid t_{0}\right)=[175,25,0,0,]^{\top}, \hat{\boldsymbol{x}}_{s_{3}}\left(t_{0} \mid t_{0}\right)=[125,125,0,0,]^{\top}, \hat{\boldsymbol{x}}_{s_{4}}\left(t_{0} \mid t_{0}\right)=$ $[-225,125,0,0,]^{\top}$, and the corresponding initial estimation error covariances were $\mathbf{P}_{r}(0 \mid 0)=\mathbf{P}_{s_{i}}(0 \mid 0)=$ $\operatorname{diag}\left[1 \times 10^{4}, 1 \times 10^{4}, 1 \times 10^{4}, 1 \times 10^{2}\right], i=2,3,4$. For meaningful comparison between the different receiver trajectories, the process and observation noise generators were primed with the same seed in all five simulations to produce the same time history (realization) of noise $\left\{\boldsymbol{w}\left(t_{k}\right)\right\}_{k=0}^{N}$ and $\left\{\boldsymbol{v}\left(t_{k}\right)\right\}_{k=0}^{N}$, respectively.

Figure 2 illustrates the five resulting receiver trajectories, and Figures 3-6 show the estimation error trajectories $\tilde{x} \triangleq x-\hat{x}$ along with the $\pm 2 \sigma_{i}$ estimation error variance bounds. The following conclusions can be drawn from these results. First, the receiver moving randomly in the environment resulted in the poorest estimates, which highlights the need for optimizing the receiver trajectory. Second, while the intuitive circular trajectory yielded better estimates than the random trajectory, it still under-performed when compared to the three optimal trajectories. Third, the D-optimality criterion outclassed the A-optimality and E-optimality criteria. Fourth, it was interesting to note that the trajectory resulting from the D-optimality criterion started by circling the anchor SOP before going in a path around the entire set of SOPs. By doing so, the receiver gained good estimates about its own states very fast, which enabled it to estimate the other unknown SOPs in the environment better. Fifth, the E-optimality resulted with the most zigzagged trajectory, which can be explained by recalling that the optimizer keeps focusing on minimizing the largest eigenvalue of the estimation error covariance matrix. So, as such eigenvalue switches its correspondence from one mode to another, the optimizer switches its "focus". Finally, all resulting trajectories exhibited the phenomenon known in SLAM as "loop closure", in which the robot (receiver in this case) deliberately "closes the loop" by revisiting already-explored areas in order to improve the overall map estimate. 


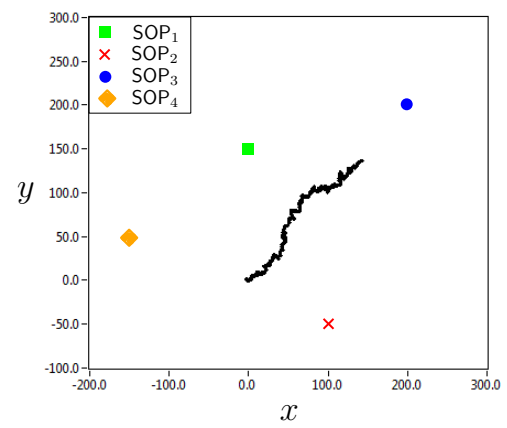

$(a)$

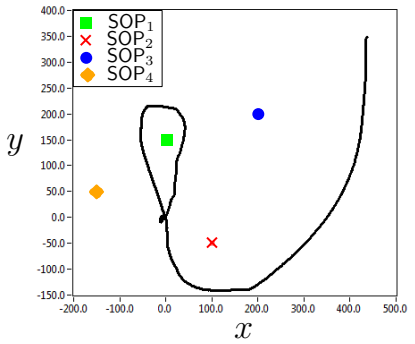

(c)

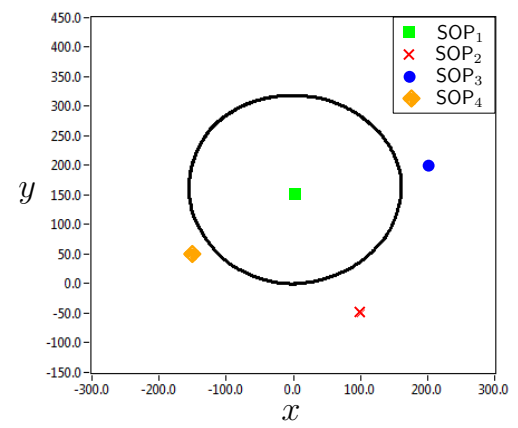

(b)

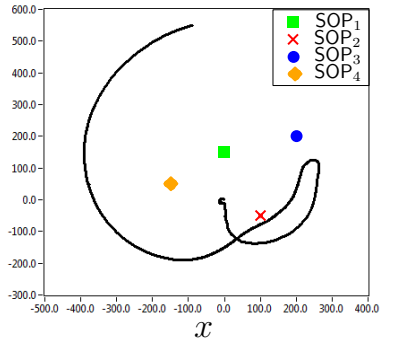

(d)

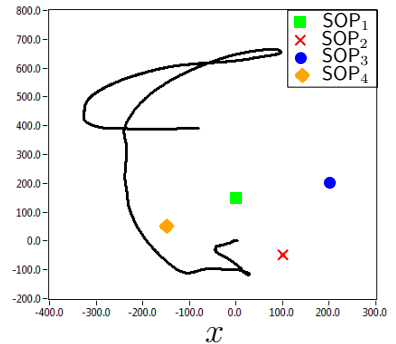

(e)

Figure 2. Receiver trajectories for $(a)$ random, $(b)$ pre-defined circular path around the fully-known anchor SOP, $(c)$ optimal path generated by the D-optimality criterion, $(d)$ optimal path generated by the A-optimality criterion, and $(e)$ optimal path generated by the E-optimality criterion
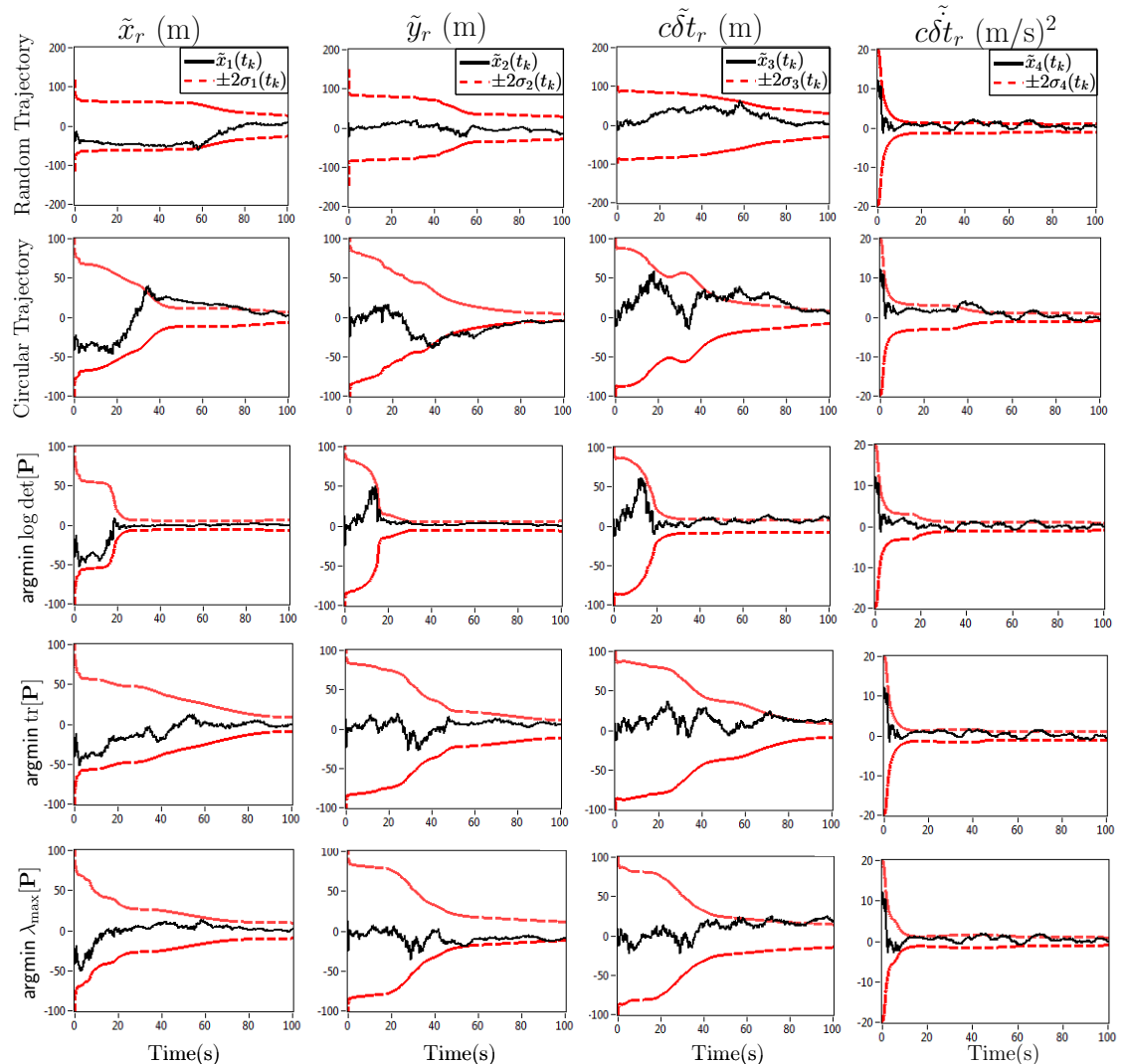

Figure 3. Receiver states estimation error trajectories for a random, pre-defined circular path around the fully-known SOP, and optimal paths generated by the D-optimality, A-optimality, and E-optimality criteria 

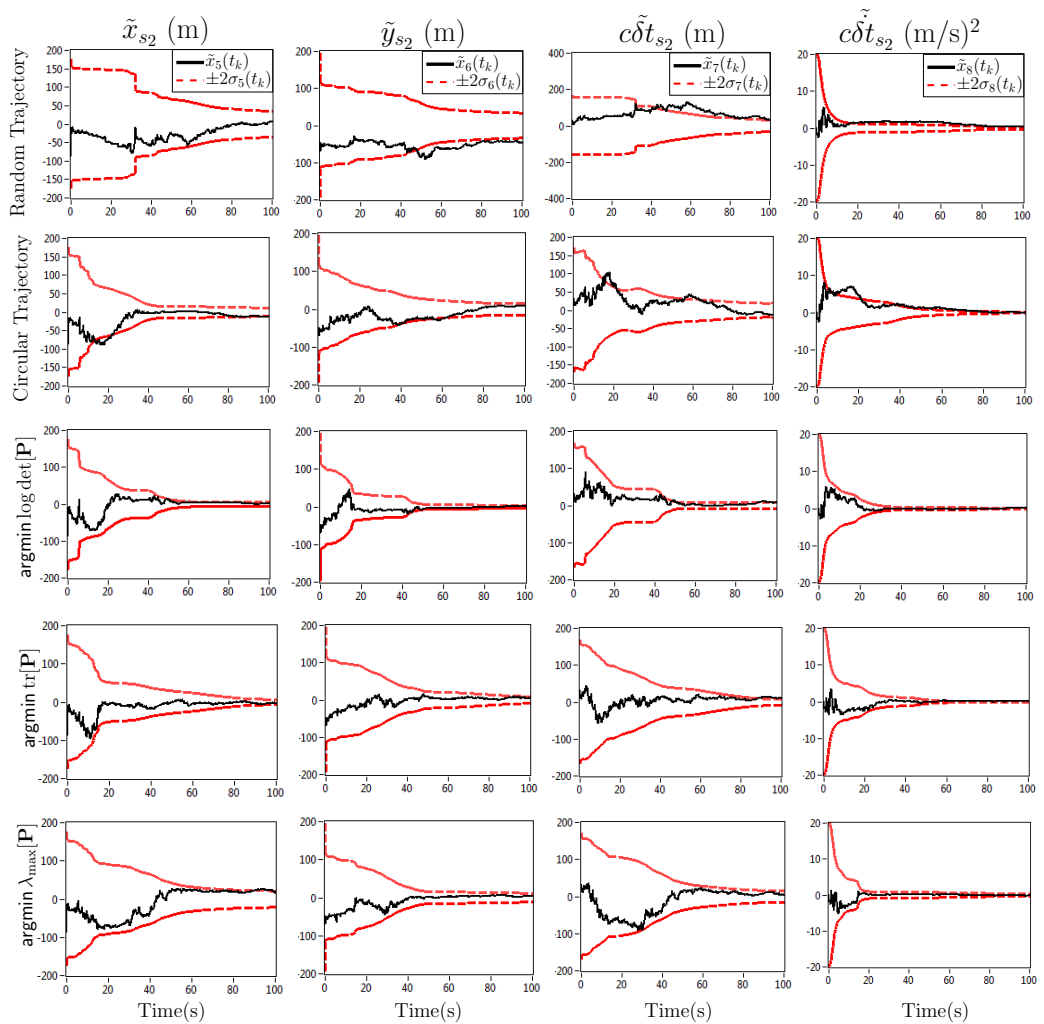

Figure 4. $\mathrm{SOP}_{2}$ states estimation error trajectories
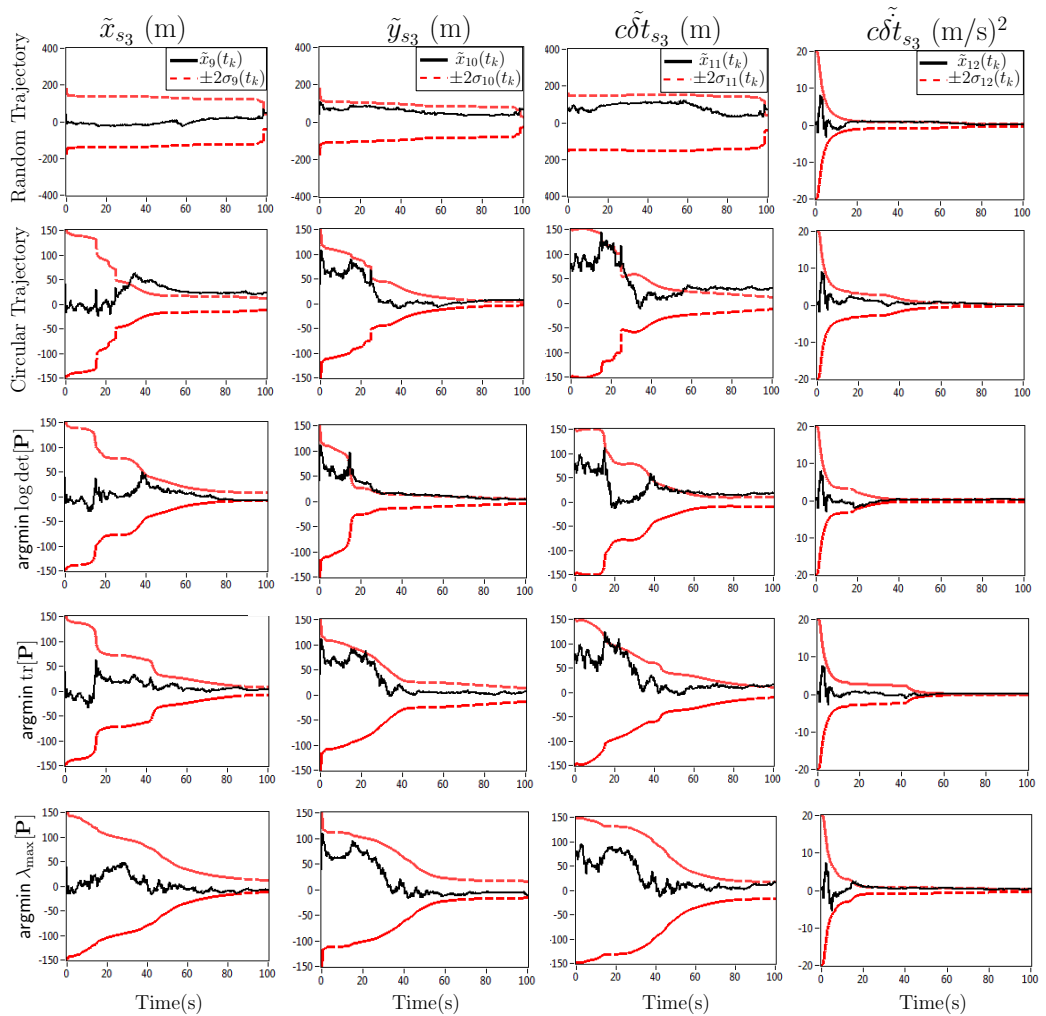

Figure 5. $\mathrm{SOP}_{3}$ states estimation error trajectories 

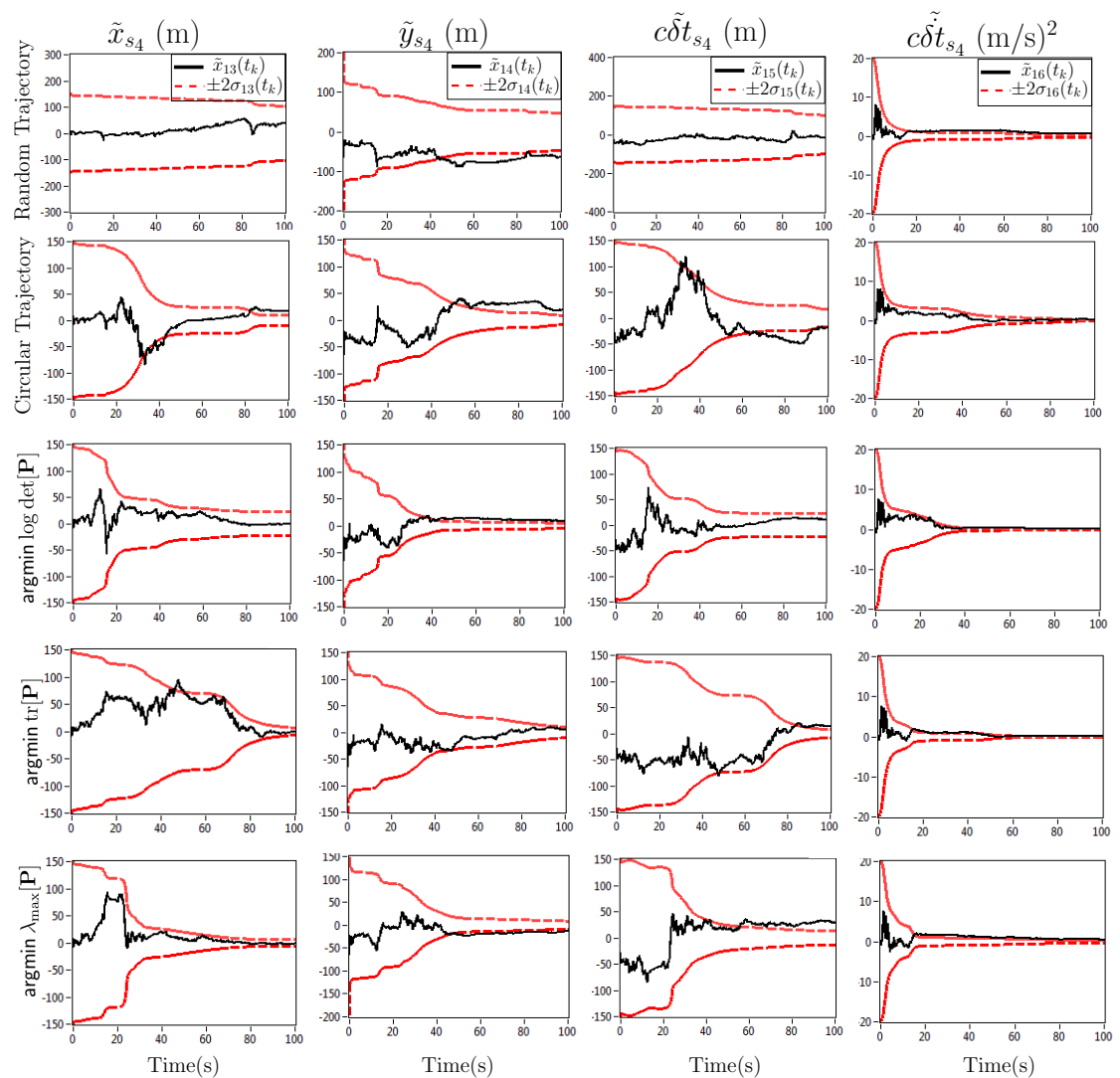

Figure 6. $\mathrm{SOP}_{4}$ states estimation error trajectories

\section{Conclusion}

This paper addressed the following problem. A receiver with no a priori knowledge about its own states is dropped in an unknown, yet observable, OpNav environment. Assuming that the receiver could prescribe its own trajectory, what motion planning strategy should the receiver adopt in order to build a high-fidelity map of the OpNav signal landscape while simultaneously localizing itself within this map in space and time? To answer this question, first, an observability analysis was conducted to establish the minimum a priori knowledge about the environment necessary for complete observability. It was shown that a controlled receiver requires less a priori knowledge about its environment to establish full observability than an uncontrolled receiver. Second, an optimal closed-loop information-theoretic strategy was proposed for receiver motion planning. Three information measures were compared: D-optimality, A-optimality, and E-optimality. It was demonstrated that all such strategies outperform a randomly moving receiver and a receiver moving in a pre-defined circular trajectory. Among the three information measures, it was demonstrated that the D-optimality criterion outclassed the A-optimality and E-optimality criteria. Future work will investigate the convexity properties of the OpNav optimization problem, if any. Moreover, while the proposed strategy prescribed the optimal local (greedy) control, future work will assess whether a multi-step look-ahead control strategy is worth the associated computational burden.

\section{Acknowledgments}

This research was partially supported by the WNCG Industrial affiliates program, and the NSF I/UCRC WICAT Center Grant IIP-1067914. 


\section{References}

${ }^{1}$ Pesyna, K., Kassas, Z., Bhatti, J., and Humphreys, T., "Tightly-coupled opportunistic navigation for deep urban and indoor positioning," Proceedings of the International Technical Meeting of the Satellite Division of the Institute of Navigation (ION GNSS), September 2011, pp. 3605-3617.

${ }^{2}$ Pesyna, K., Kassas, Z., and Humphreys, T., "Constructing a continuous phase time history from TDMA signals for opportunistic navigation," Proceedings of IEEE/ION Position Location and Navigation Symposium (PLANS), April 2012, pp. $1209-1220$

${ }^{3}$ Kassas, Z., "Collaborative opportunistic navigation," IEEE Aerospace and Electronic Systems Magazine, Vol. 28, No. 6, June 2013, pp. 38-41.

${ }^{4}$ Durrant-Whyte, H. and Bailey, T., "Simultaneous localization and mapping: part I," IEEE Robotics ES Automation Magazine, Vol. 13, No. 2, June 2006, pp. 99-110.

${ }^{5}$ Bailey, T. and Durrant-Whyte, H., "Simultaneous localization and mapping: part II," IEEE Robotics 83 Automation Magazine, Vol. 13, No. 3, September 2006, pp. 108-117.

${ }^{6}$ Kassas, Z. and Humphreys, T. E., "Observability analysis of opportunistic navigation with pseudorange measurements," Proceedings of AIAA Guidance, Navigation, and Control Conference (GNC), August 2012, pp. 4760-4775.

${ }^{7}$ Kassas, Z. and Humphreys, T., "Observability and estimability of collaborative opportunistic navigation with pseudorange measurements," Proceedings of the International Technical Meeting of the Satellite Division of the Institute of Navigation (ION GNSS), September 2012, pp. 621-630.

${ }^{8}$ Oshman, Y. and Davidson, P., "Optimization of observer trajectories for bearings-only target localization," IEEE Transactions on Aerospace and Electronic Systems, Vol. 35, No. 3, July 1999, pp. 892-902.

${ }^{9}$ Frew, E. W., "Receding horizon control using random search for UAV navigation with passive, non-cooperative sensing," Proceedings of AIAA Guidance, Navigation, and Control Conference (GNC), August 2005, pp. 5864-5876.

${ }^{10}$ Ponda, S. S., Kolacinski, R. M., and Frazzoli, E., "Trajectory optimization for target localization using small unmanned aerial vehicles," Proceedings of AIAA Guidance, Navigation, and Control Conference (GNC), August 2009, pp. 1209-1220.

${ }^{11}$ Feder, H., Leonard, J., and Smith, C., "Adaptive mobile robot navigation and mapping," International Journal of Robotics Research, Vol. 18, No. 7, July 1999, pp. 650-668.

${ }^{12}$ Leung, C., Huang, S., Kwok, N., and Dissanayake, G., "Planning under uncertainty using model predictive control for information gathering," Robotics and Autonomous Systems, Vol. 54, No. 11, November 2006, pp. 898-910.

${ }^{13}$ Lidoris, G., Kuhnlenz, K., Wollherr, D., and Buss, M., "Combined trajectory planning and gaze direction control for robotic exploration," Proceedings IEEE International Conference on Robotics and Automation, April 2007, pp. 4044-4049.

${ }^{14}$ Cochran, W., "Experiments for nonlinear functions," Journal of the American Statistical Association, Vol. 68, No. 344, December 1973, pp. 771-781.

${ }^{15}$ Thompson, A., Moran, J., and Swenson, G., Interferometry and Synthesis in Radio Astronomy, John Wiley, 2nd ed., 2001.

${ }^{16}$ Bar-Shalom, Y., Li, X., and Kirubarajan, T., Estimation with Applications to Tracking and Navigation, John Wiley, New York, NY, 2002.

${ }^{17}$ Brown, R. and Hwang, P., Introduction to Random Signals and Applied Kalman Filtering, John Wiley, 3rd ed., 2002.

${ }^{18}$ Psiaki, M. and Mohiuddin, S., "Modeling, analysis, and simulation of GPS carrier phase for spacecraft relative navigation," Journal of Guidance, Control, and Dynamics, Vol. 30, No. 6, November-December 2007, pp. 1628-1639.

${ }^{19}$ Anguelova, M., Observability and identifiability of nonlinear systems with applications in biology, Ph.D. thesis, Chalmers University Of Technology and Göteborg University, Sweden, 2007.

${ }^{20}$ Casti, J., "Recent developments and future perspectives in nonlinear system theory," SIAM Review, Vol. 24, No. 3, July 1982, pp. 301-331.

${ }^{21}$ Hermann, R. and Krener, A., "Nonlinear controllability and observability," IEEE Transactions on Automatic Control, Vol. 22, No. 5, October 1977, pp. 728-740.

${ }^{22}$ Respondek, W., "Geometry of static and dynamic feedback," Lecture Notes at the Summer School on Mathematical Control Theory, Trieste, Italy, September 2001.

${ }^{23}$ Durrant-Whyte, H., Multi Sensor Data Fusion, 2001.

${ }^{24}$ Cover, T. and Thomas, J., Elements of Information Theory, Wiley-Interscience, 2nd ed., 2006.

${ }^{25}$ Uciński, D., Optimal Measurement Methods for Distributed Parameter System Identification, CRC Press, 2005. 